\title{
Peak Lag Between Plasma Vasopressin and Urine Aquaporin-2 Following Cardiac Surgery
}

\author{
Shigeki Yokoyama, ${ }^{1} \mathrm{MD}$, Teruhiko Imamura, ${ }^{2} \mathrm{MD}$, Shigeyuki Yamashita, ${ }^{1} \mathrm{MD}$, Toshio Doi, ${ }^{1} \mathrm{MD}$, \\ Kazuaki Fukahara, ${ }^{1}$ MD, Naoki Yoshimura, ${ }^{1}$ MD and Koichiro Kinugawa, ${ }^{2}$ MD
}

\begin{abstract}
Summary
Tolvaptan, a vasopressin type- 2 receptor antagonist, is utilized to ameliorate fluid retention following cardiac surgery. However, the optimal timing of tolvaptan administration considering novel biomarkers remains unknown. We prospectively included patients who underwent cardiac surgery between 2016 and 2020. We measured perioperative trends of free water reabsorption mediators including plasma arginine vasopressin and urine aquaporin-2. A total of 20 patients $(68[60,75]$ years old, $18 \mathrm{men})$ were included. Urine volume decreased gradually after the initial 3 hours following cardiac surgery. The plasma arginine vasopressin level increased significantly with a peak at postoperative 6 hours, whereas the urine aquaporin-2 level increased later with a delayed peak at postoperative 12 hours. As a result, urine aquaporin-2 relative to the plasma arginine vasopressin level, which represents the activity of the collecting ducts and indicates predicted responses to tolvaptan, was a minimum at postoperative 6 hours. Tolvaptan administration immediately after cardiac surgery might not be recommended given the transient refractoriness to tolvaptan probably due to the stunning of kidney collecting ducts.
\end{abstract}

(Int Heart J Advance Publication)

Key words: Heart failure, Hemodynamics, Diuretics, Tolvaptan

$\mathrm{D}$ espite the improvement in perioperative clinical management and surgical technique, fluid overload is one of the critical issues associating with mortality and morbidity following cardiac surgery. ${ }^{1}$ Loop diuretics are a conventional therapeutic tool to treat postoperative fluid overload, ${ }^{2)}$ but are associated with poor clinical outcomes owing to the stimulations of sympathetic nerve activity and renin-angiotensin-aldosterone system, progression of hyponatremia, hypotension, and reactive tachycardia, if their doses were inappropriately high. ${ }^{1)}$

Tolvaptan, a vasopressin type- 2 receptor antagonist, is a recently-introduced novel diuretic that suppresses reabsorption of free water in the kidney collecting duct and increases urine output, relatively maintaining hemodynamics and renal function and improving quality of life in various clinical situations accompanying fluid overload. ${ }^{2}$

Recent studies have demonstrated the clinical implications of administering tolvaptan peri-operatively in patients receiving cardiac surgery in improving postoperative clinical outcomes by ameliorating fluid overload.-8) However, the optimal timing to administer tolvaptan remains uncertain (i.e., prior to the surgery, immediately after the surgery, or several hours following the surgery).

In patients with heart failure, the plasma arginine vasopressin (AVP) level is inappropriately elevated due to reduced cardiac output via a non-osmotic pathway." Increased AVP stimulates re-absorption of free water in the kidney collecting duct via activated and increased aquaporin-2 (AQP2). ${ }^{10)}$ In responders to tolvaptan, urine AQP2 relative to the plasma AVP level is appropriately high. ${ }^{11)}$ In non-responders, the urine AQP2 level is low despite an increased plasma AVP level due to impaired function of the kidney collecting ducts. Thus, urine AQP2 relative to the plasma AVP level is an established predictor of responses to tolvaptan. ${ }^{11}$

Given the drastic changes in hemodynamics following cardiac surgery, the response to tolvaptan might change dynamically. Trend analyses of these waterreabsorption mediators would clarify the optimal timing of tolvaptan administration. In this study, we investigated the trends of plasma AVP and urine AQP2 following cardiac surgery in order to clarify the optimal timing of tolvaptan administration.

\section{Methods}

Study design: Patients who underwent scheduled cardiac surgery by the attending cardiac surgeon (SY) and gave written informed consent at our institute between 2016 and 2020 were prospectively included in this study. Pa-

From the ${ }^{1}$ Department of Surgery 1, Faculty of Medicine, University of Toyama, Toyama, Japan and ${ }^{2}$ Second Department of Internal Medicine, University of Toyama, Toyama, Japan.

Address for correspondence: Koichiro Kinugawa, MD, The Second Department of Internal Medicine, University of Toyama, 2630 Sugitani, Toyama, Toyama 930-0194, Japan. E-mail: kinugawa-tky@umin.ac.jp

Received for publication May 14, 2021. Revised and accepted June 28, 2021.

Released in advance online on J-STAGE September 17, 2021.

doi: $10.1536 /$ ihj.21-301

All rights reserved by the International Heart Journal Association. 
Table. Baseline Characteristics

\begin{tabular}{lc}
\hline Variable & \\
\hline Demographics & \\
Age, years & $68(60,75)$ \\
Men & $18(90 \%)$ \\
Body weight, kg & $64.4(58.0,79.5)$ \\
Ischemic heart disease & $12(60 \%)$ \\
Diabetes mellitus & $6(30 \%)$ \\
Atrial fibrillation & $5(25 \%)$ \\
Operation data & \\
Operation type & \\
Off-pump coronary artery bypass grafting & $8(40 \%)$ \\
Valve surgery & $9(45 \%)$ \\
Valve surgery and coronary artery bypass grafting & $3(15 \%)$ \\
Operation time, min & $193(179,220)$ \\
Cardiopulmonary bypass time min $(n=12)$ & $134(122,145)$ \\
Postoperative transfusion, mL $(n=4)$ & $990(540,1440)$ \\
Laboratory and echocardiography & \\
Hemoglobin, g/dL & $15.0(13.9,16.1)$ \\
Serum albumin, g/dL & $4.2(4.1,4.3)$ \\
eGFR, mL/minute/1.73 m ${ }^{2}$ & $67.6(58.8,75.5)$ \\
Plasma arginine vasopressin, pg/mL & $1.7(1.2,3.5)$ \\
Urine aquaporin- 2, ng/mL & $6.4(2.1,9.9)$ \\
Plasma NTpro-B-type natriuretic peptide, pg/mL & $483(157,1077)$ \\
Left ventricular ejection fraction, $\%$ & $65(47,70)$ \\
\hline Continuous variables are expressed as the median and interquartile and categorical vari- \\
ables as a number and percentage. eGFR indicates estimated glomerular filtration ratio.
\end{tabular}

tients dependent on hemodiafiltration and those with an estimated glomerular filtration ratio $<30 \mathrm{~mL} /$ minute $/ 1.73$ $\mathrm{m}^{2}$ were excluded. The study protocol was approved by the local ethics committee.

Operative technique and perioperative management: All patients underwent cardiac surgery via a midsternotomy incision in a standard manner. The cardiopulmonary bypass system was established by the standard technique and performed at moderate hypothermia (30$32^{\circ} \mathrm{C}$ ). Myocardial protection during the operation included intermittent antegrade cold blood cardioplegia. The off-pump coronary artery bypass grafting surgery was performed at normothermia. Postoperative management was performed according to standard cardiac surgery postoperative management. Diuretics including furosemide were not administered in any patient perioperatively.

Data collection: The baseline characteristics of the patients including demographic data within 24 hours before the cardiac surgery and operation data were collected. Plasma AVP and urine AQP2 levels were measured within 24 hours before the cardiac surgery as baseline variables.

Following the cardiac surgery, urine volumes were measured every 1 hour during the initial 24 hours. Plasma AVP and urine AQP2 levels were measured at postoperative 6 hours, 12 hours, and 1 week.

Statistical analyses: Continuous variables are presented as the median and interquartile. Categorical variables are presented as numbers and percentages. Trends were assessed by using the Friedman test and ad-hoc Wilcoxon signed-rank test versus baseline values. The response to tolvaptan was predicted by urine AQP2/plasma AVP, which indicates the residual ability of the kidney collect- ing ducts to increase AQP2 by responding to the stimulation of AVP. ${ }^{11)}$ Statistical analyses were performed using SPSS Statistics 22 (SPSS Inc, Armonk, IL, USA). Twosided $P$-values $<0.05$ were considered statistically significant.

\section{Results}

Baseline characteristics: Twenty patients (68 [60, 75] years old, 18 men) were included. Among them, 8 underwent off-pump coronary artery bypass grafting, 9 valve surgery, and 3 valve surgery and coronary artery bypass grafting. The baseline characteristics are summarized in the Table.

Trends of urine volume: Trends of urine volume during the initial 24 hours following cardiac surgery are summarized in Figure 1. The urine volume per hour gradually decreased with a peak at postoperative 2 hours $(P<$ 0.001).

Perioperative trends of urine volume mediators: Following cardiac surgery, the plasma AVP level increased significantly with a peak value at postoperative 6 hours $(P$ $<0.05$; Figure 2A). The urine AQP2 level increased gradually following cardiac surgery with a peak value at postoperative 12 hours $(P<0.05$; Figure $2 \mathrm{~B})$. Urine osmolality tended to increase following the cardiac surgery with a peak at postoperative 12 hours (Figure 2C).

As a result, a plasma AVP peak is located at 6 hours (red arrow in Figure 1), followed by a urine APQ2 peak at 12 hours (blue arrow in Figure 1), as well as gradual decreases in urine volume after the initial 6 hours.

Perioperative trend of estimated responses to tolvap- 


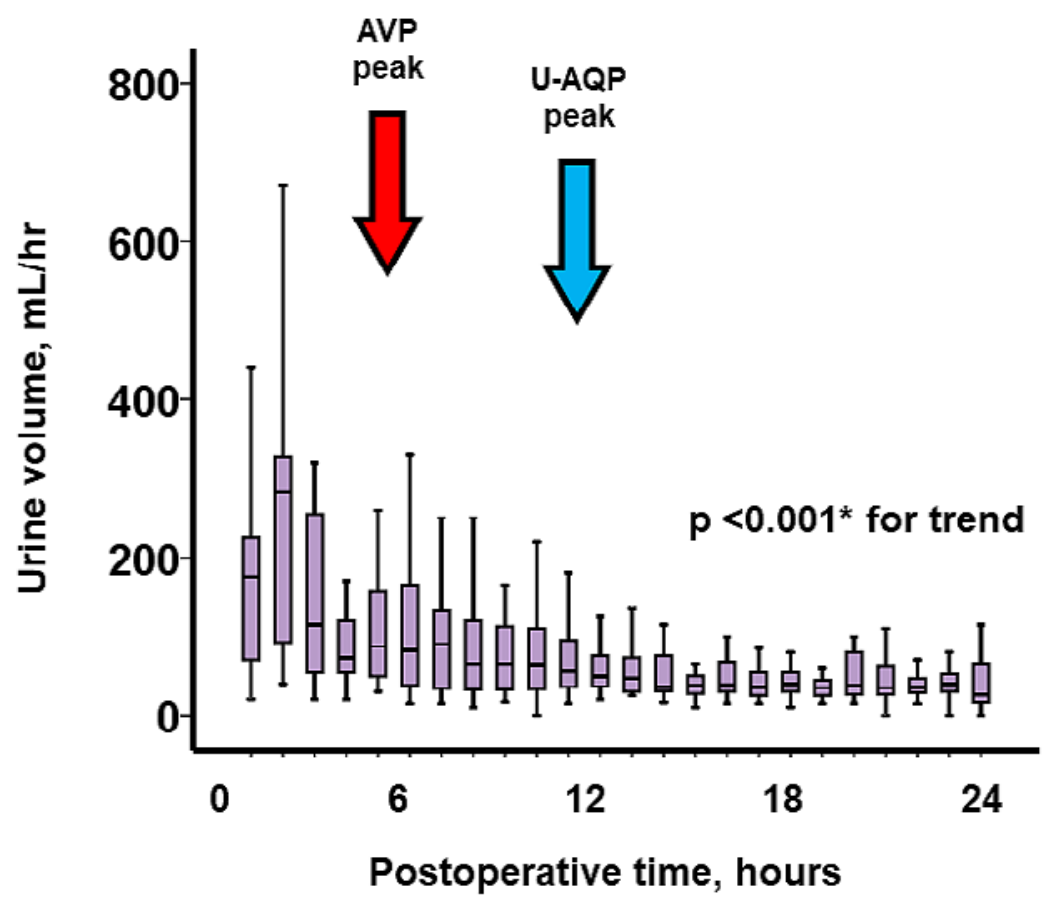

Figure 1. Trend in urine volume during 24 hours following cardiac surgeries. AVP indicates arginine vasopressin; and $\mathrm{U}-\mathrm{AQP}$, urine aquaporin. $* P<0.05$ by Friedman test.

A

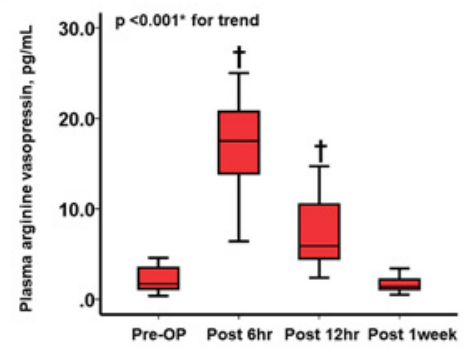

B

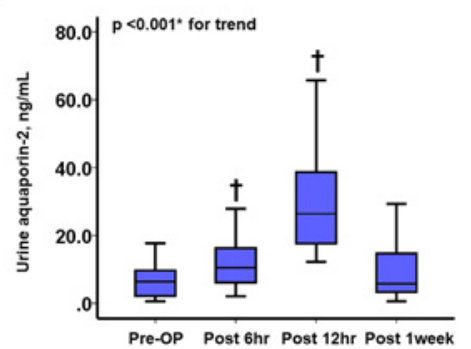

C

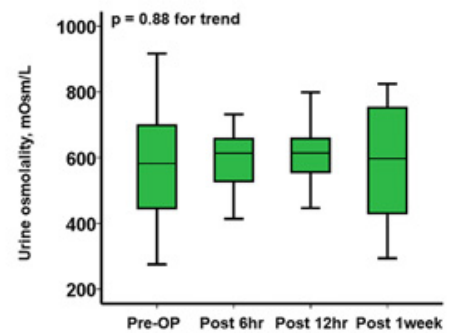

Figure 2. Perioperative trends in plasma arginine vasopressin $(\mathbf{A})$, urine aquaporin-2 (B), and urine osmolality $(\mathbf{C})$. $* P<0.05$ by Friedman test. ${ }^{\dagger} P<0.05$ by post-hoc Mann-Whitney $U$ test versus preoperative value.

tan: At baseline, median urine AQP2/plasma AVP, which indicated preserved kidney collecting duct function and was an estimated ability to respond to tolvaptan, was 2.4 $(1.3,4.5) \times 10^{3}$ (Figure 3$)$. All patients had values above $0.5 \times 10^{3}$, which was the cutoff for responders proposed in a previous study. ${ }^{11)}$ Following the surgery, the value dropped transiently at postoperative 6 hours down to 0.6 $(0.3,1.2) \times 10^{3}(P<0.05)$ and recovered at 12 hours.

Urine AQP2/plasma AVP levels at postoperative 6 hours were statistically not different between those with cardiopulmonary bypass $(n=12)$ and those without $(n=$ 8) $\left(0.5[0.3,0.9]\right.$ versus $\left.1.1[0.4,1.3] \times 10^{3}, P=0.34\right)$ and between those with transfusion $(n=4)$ and those without $(n=16)(1.1[0.6,1.6]$ versus $0.5[0.3,1.1] \times$ $\left.10^{3}, P=0.49\right)$.

\section{Discussion}

In this prospective study, we investigated the trends of free water reabsorption mediators including plasma AVP and urine AQP2 following cardiac surgery. A peak of plasma AVP was observed at 6 hours whereas that of urine AQP2 was observed at 12 hours when urine volume per hour was consistently lower. Urine AQP2 level relative to plasma AVP was preserved in all patients at baseline (> $\left.0.5 \times 10^{3}\right)$, decreasing transiently at 6 hours and recovering at 12 hours.

Fluid retention following cardiac surgery: Fluid retention is often encountered following cardiac surgery and is associated with mortality and morbidity. ${ }^{1)}$ Hemodilution due to cardiopulmonary bypass stimulates the osmotic pathway and increases the plasma AVP level. ${ }^{12)}$ Reperfusion injures the myocardium and provokes transient heart failure, which stimulates non-osmotic pathways and also 


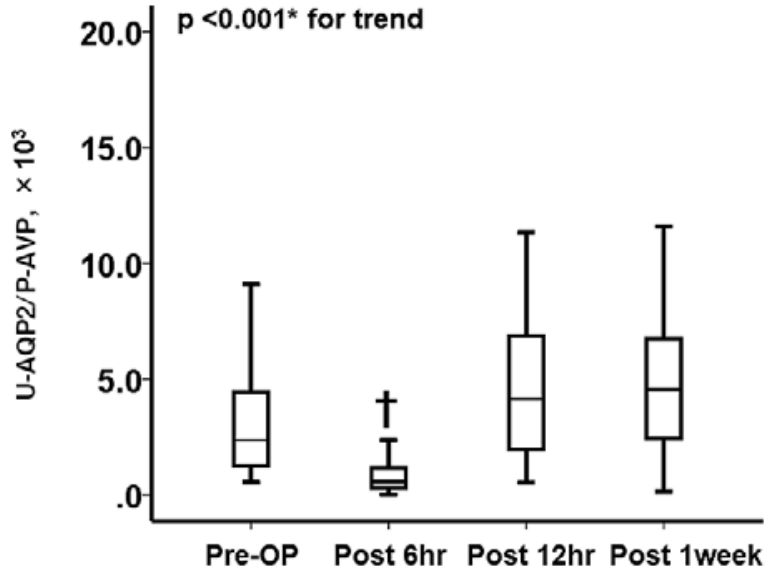

Figure 3. Perioperative trends in urine aquaporin-2/plasma arginine vasopressin. The value dropped transiently at postoperative 6 hours and recovered up to the preoperative levels at 12 hours. $* P<0.05$ by Friedman test. ${ }^{\dagger} P<0.05$ by post-hoc Mann-Whitney $U$ test versus preoperative value.

increases plasma AVP levels. ${ }^{13)}$ Increased plasma AVP stimulates vasopressin type- 2 receptors located on the kidney collecting ducts and facilitates reabsorption of free water via activating the AQP2 system. ${ }^{10)}$ Perioperative inflammation increases vascular permeability and worsens interstitial edema. ${ }^{12)}$ Given these mechanisms, tolvaptan, a vasopressin type- 2 receptor antagonist, has received a lot of attention with respect to its ability to possibly correct postoperative fluid retention.

Perioperative concomitant tolvaptan therapy: Several studies have demonstrated that tolvaptan concomitant therapy was associated with higher urine output, earlier body weight recovery, and shorter hospitalization length compared with a conventional loop diuretic therapy in patients receiving cardiac surgery. ${ }^{3-8)}$ A reno-protective effect of tolvaptan therapy was also observed in some of them., ${ }^{4,8}$ Tolvaptan seems to have been administered immediately following the cardiac surgery in most of the studies. However, the theoretically and clinically optimal timing of tolvaptan therapy has not been discussed.

Optimal timing of tolvaptan administration: In general, the urine AQP2 level should have a positive correlation with the plasma AVP level when kidney collecting duct function is preserved. ${ }^{11)}$ In this study, we observed a "time lag" between the peak of plasma AVP (at postoperative 6 hours) and the peak of urine AQP2 (at postoperative 12 hours). Consistently, urine AQP2 level relative to plasma AVP, which indicates the residual function of the kidney collecting ducts and a predictor of the response to tolvaptan, decreased transiently at 6 hours and normalized at 12 hours.

Given that tolvaptan increases urine volume by suppressing the activity of the AQP2 system, ${ }^{10)}$ the optimal timing of tolvaptan administration might be relatively later instead of the postoperative immediate phase that most of the studies adopted. Such knowledge should be useful, particularly several years later when intra-venous tolvaptan will be clinically available.

The detailed mechanism that explains the time lag re- mains unknown, but the inflammatory injury of kidney interstitial tissue by the invasion of cardiac surgery including cardiopulmonary bypass might transiently suppress the activity of the kidney collecting ducts at the postoperative immediate phase. ${ }^{14)}$

Another concern is optimal patient selection. Previous studies demonstrated that a decrease in urine osmolality following the administration of tolvaptan and a shorter cardiopulmonary bypass time were associated with a good response to tolvaptan among those receiving cardiac surgery. ${ }^{3,4,7,8)}$ In our study, all patients satisfied urine AQP2/plasma AVP $>0.5 \times 10^{3}$ at baseline, which is another cutoff for responders to tolvaptan. ${ }^{11}$

Limitations: The sample size is small. We observed a time lag between plasma AVP and urine AQP2 but the mechanism to explain this phenomenon remains within the realm of speculation. We did not use any diuretics following the cardiac surgery, and our findings might not simply be applicable to those who receive diuretics perioperatively.

We did not analyze the response to tolvaptan by actually administering tolvaptan, which should be assessed in subsequent prospective randomized control trials comparing early tolvaptan therapy (at 6 postoperative hours) and late tolvaptan therapy (at postoperative 12 hours).

\section{Conclusion}

The plasma arginine level increased following cardiac surgery, whereas urine aquaporin-2, which is a marker of the responder to tolvaptan, increased later with a delayed peak. Tolvaptan administration immediately after cardiac surgery might not be effective given transient stunning of the kidney collecting ducts.

\section{Disclosure}

Conflicts of interest: TI receives grant support from JSPS KAKENHI: JP20K17143.

Data availability: The data that support the findings of this study are available from the corresponding author upon reasonable request.

\section{References}

1. Stein A, de Souza LV, Belettini CR, Menegazzo WR, Viegas JR, Costa Pereira EM, et al. Fluid overload and changes in serum creatinine after cardiac surgery: predictors of mortality and longer intensive care stay. A prospective cohort study. Crit Care 2012; 16: R99.

2. Imamura T, Kinugawa K. Update of acute and long-term tolvaptan therapy. J Cardiol 2019; 73: 102-7.

3. Ito H, Mizumoto T, Tempaku H, Fujinaga K, Sawada Y, Shimpo H. Efficacy of Tolvaptan on Fluid Management After Cardiovascular Surgery Using Cardiopulmonary Bypass. J Cardiothorac Vasc Anesth 2016; 30: 1471-8.

4. Yamada M, Nishi H, Sekiya N, Horikawa K, Takahashi T, Sawa $\mathrm{Y}$. The efficacy of tolvaptan in the perioperative management of chronic kidney disease patients undergoing open-heart surgery. Surg Today 2017; 47: 498-505.

5. Hagiwara S, Nishida N, Chishina H, et al. Cases with Refractory Ascites and a Delayed Response to Tolvaptan. Intern Med 
2016; 55: 3273-7

6. Nishi H, Toda K, Miyagawa S, et al. Effects of tolvaptan in the early postoperative stage after heart valve surgery: results of the STAR (Study of Tolvaptan for fluid retention AfteR valve surgery) trial. Surg Today 2015; 45: 1542-51.

7. Kishimoto Y, Nakamura Y, Harada S, et al. Can Tolvaptan Protect Renal Function in the Early Postoperative Period of Cardiac Surgery?- Results of a Single-Center Randomized Controlled Study. Circ J 2018; 82: 999-1007.

8. Matsuyama K, Koizumi N, Nishibe T, et al. Effects of shortterm administration of tolvaptan after open heart surgery. Int $\mathrm{J}$ Cardiol 2016; 220: 192-5.

9. Imamura T, Kinugawa K, Hatano M, et al. Low cardiac output stimulates vasopressin release in patients with stage $d$ heart failure. Circ J 2014; 78: 2259-67.

10. Imamura $\mathrm{T}$, Kinugawa K. Urine Aquaporin-2: A Promising
Marker of Response to the Arginine Vasopressin Type-2 Antagonist, Tolvaptan in Patients with Congestive Heart Failure. Int J Mol Sci 2016; 17: 105.

11. Imamura $\mathrm{T}$, Kinugawa $\mathrm{K}$, Fujino $\mathrm{T}$, et al. Increased urine aquaporin-2 relative to plasma arginine vasopressin is a novel marker of response to tolvaptan in patients with decompensated heart failure. Circ J 2014; 78: 2240-9.

12. Jochberger S, Mayr VD, Luckner G, et al. Serum vasopressin concentrations in critically ill patients. Crit Care Med 2006; 34: 293-9.

13. Kaul TK, Swaminathan R, Chatrath RR, Watson DA. Vasoactive pressure hormones during and after cardiopulmonary bypass. Int J Artif Organs 1990; 13: 293-9.

14. Kumar AB, Suneja M. Cardiopulmonary bypass-associated acute kidney injury. Anesthesiology 2011; 114: 964-70. 